\title{
Special functions, integral equations and Riemann-Hilbert problem
}

\author{
R. Wong ${ }^{a}$ and Yu-Qiu Zhao ${ }^{b}$ \\ ${ }^{a}$ Department of Mathematics, City University of Hong Kong, Kowloon, Hong Kong \\ ${ }^{b}$ Department of Mathematics, Sun Yat-sen University, GuangZhou 510275, China
}

\begin{abstract}
We consider a pair of special functions, $u_{\beta}$ and $v_{\beta}$, defined respectively as the solutions to the integral equations

$$
u(x)=1+\int_{0}^{\infty} \frac{K(t) u(t) d t}{t+x} \text { and } v(x)=1-\int_{0}^{\infty} \frac{K(t) v(t) d t}{t+x}, \quad x \in[0, \infty),
$$

where $K(t)=\frac{1}{\pi} \exp \left(-t^{\beta} \sin \frac{\pi \beta}{2}\right) \sin \left(t^{\beta} \cos \frac{\pi \beta}{2}\right)$ for $\beta \in(0,1)$. In this note, we establish the existence and uniqueness of $u_{\beta}$ and $v_{\beta}$ which are bounded and continuous in $[0,+\infty)$. Also, we show that a solution to a model Riemann-Hilbert problem in Kriecherbauer and McLaughlin [Int. Math. Res. Not., 1999] can be constructed explicitly in terms of these functions. A preliminary asymptotic study is carried out on the Stokes phenomena of these functions by making use of their connection formulas.

Several open questions are also proposed for a thorough investigation of the analytic and asymptotic properties of the functions $u_{\beta}$ and $v_{\beta}$, and a related new special function $G_{\beta}$.
\end{abstract}

Keywords: Special function; Freud weight; integral equation; Riemann-Hilbert problem; asymptotics; Stokes phenomenon.

Mathematics Subject Classification 2010: 33E30, 41A60, 45A05

\section{Introduction}

Kriecherbauer and McLaughlin [8] used the Riemann-Hilbert approach to study the strong asymptotics of the polynomials orthogonal with respect to the Freud weight

$$
w_{\beta}(x) d x=e^{-\kappa_{\beta}|x|^{\beta}} d x, \quad x \in \mathbb{R}, \quad \kappa_{\beta}=\frac{\Gamma\left(\frac{\beta}{2}\right) \Gamma\left(\frac{1}{2}\right)}{\Gamma\left(\frac{\beta+1}{2}\right)}, \quad \beta>0 .
$$

When $0<\beta<1$, several representative results are obtained in terms of a model RiemannHilbert problem (RHP) for a certain $2 \times 2$ matrix-valued function $L$ :

$$
\begin{aligned}
& L: \mathbb{C} \backslash \mathbb{R} \rightarrow \mathbb{C}^{2 \times 2} \text { is analytic, } \\
& L_{+}(s)=L_{-}(s) v_{L}(s) \text { for } s \in \mathbb{R}, \\
& L(s)=O(\ln |s|) \text { as } s \rightarrow 0, \\
& L(s) \rightarrow I \text { for } s \rightarrow \infty,
\end{aligned}
$$


where the jump matrix is given by

$$
v_{L}(s)=\left(\begin{array}{cc}
1 & -\eta_{L}(s) \\
\eta_{L}(-s) & 1
\end{array}\right), s \in \mathbb{R}
$$

with

$$
\eta_{L}(s)=2 i(-1)^{n+1} e^{-|s|^{\beta} \sin (\pi \beta / 2)} \sin \left(|s|^{\beta} \cos \frac{\pi \beta}{2}\right) \mathbf{1}_{[0, \infty)}(s), s \in \mathbb{R},
$$

and $n$ being the polynomial degree; see [8, (6.41)-(6.43)].

Later in this note, we will give a more natural description of the behavior of $L(s)$ at the origin. Instead of (3), we henceforth assume

$$
L(s)=O\left(\epsilon_{\beta}(s)\right) \quad \text { as } s \rightarrow 0, \quad \epsilon_{\beta}(s)= \begin{cases}1, & 1 / 2<\beta<1, \\ \ln |s|, & \beta=1 / 2, \\ |s|^{\beta-1 / 2}, & 0<\beta<1 / 2 .\end{cases}
$$

Remark 1. The uniqueness of the solution to the RHP for L, subject to either (3) or (5), can be justified by using Liouville's theorem. Now let $L_{33}(s)$ be the solution to the RHP with local behavior (3), and $L_{5}(s)$ be that with local behavior (5), then $L_{3}(s) L_{5}^{-1}(s)$ has no jump on the real line $\mathbb{R}$, and has only an isolated singularity at the origin. Since the singularity at the origin is not a pole or an essential singularity, it must be removable. In addition, since $L_{[3}(s) L_{5}^{-1}(s)$ approaches $I$ as $s \rightarrow \infty$, by Liouville's theorem this matrix-valued entire function is the identity matrix. Thus $L_{3}(s) \equiv L_{5}(s)$ if both solutions exist. In view of Lemma 圆 below, the local behavior in (5) is in a sense more natural. Later in Section 4, we will see from Theorem 3 that we actually have $L(s)=O(1)$ as $s \rightarrow 0$ for the parametrix constructed in (13). Accordingly, both (3) and (5) are fulfilled.

In [8], the existence and uniqueness of $L$ were established in the Wiener class. For $0<\beta<1$, the leading order behavior of the solution to the model problem at the origin has not been determined explicitly; see the comment in Deift et al. [5, p.62, Remark 3.6]. For a brief historical account of the asymptotics of the orthogonal polynomials associated with the Freud weight, the reader is referred to [8] and the references therein.

Recently, there are other orthogonal polynomials with weights having similar singularities as the Freud weight. For example, Chen and Ismail [2] considered the Freud-like orthogonal polynomials arising from a recurrence relation. The polynomials are related to the indeterminate moment problems. The weight function, supported on $\mathbb{R}$, has a singularity at 0 which is similar to the Freud weight (11); see also [3, (1.6)] for the weight, and for a difference equation approach to obtain the Plancherel-Rotach asymptotics.

In [4, Deaño, Kuijlaars and Román consider the zero distribution of polynomials orthogonal with respect to the Bessel function $J_{\nu}(x)$. They encountered a varying exponential weight of Freud type $e^{n \pi|x|}$ with a potential function $\pi|x|$. To justify the existence of a certain local parametrix, a quite unnatural restriction on $\nu$ has to be brought in. Again, the leading order behavior of the parametrix has not been explicitly given.

To solve those parametrices and model problems, one may either represent them in terms of known special functions, or alternatively use new special functions to serve the same purpose.

The objective of the present note is to introduce a pair of special functions, $u_{\beta}$ and $v_{\beta}$, determined by two linear integral equations. Based on these functions, the solution to the model problem of Kriecherbauer and McLaughlin [8] can readily be constructed. 
In the rest of this note, we will address the unique solvability of the integral equations in $C[0, \infty)$, derive the behavior of the specific solutions $u_{\beta}$ and $v_{\beta}$ at infinity and at the origin, and carry out a brief discussion of the Stokes phenomena of these functions, taking the integral equations as resurgent relations. We will also propose a thorough investigation of the analytic and asymptotic properties of the new special functions $u_{\beta}$ and $v_{\beta}$, and a relevant new special function $G_{\beta}$.

\section{Integral equations and the $\mathrm{RH}$ problem}

First, let us derive the integral equations from the model problem for $L$ formulated in Section 1. From the jump condition (2) and the behavior (44)-(5), it is readily seen that the $(1,1)$-entry $L_{11}(s)$ is analytic in $\mathbb{C} \backslash(-\infty, 0]$, such that

$$
\begin{aligned}
& \left(L_{11}\right)_{+}(s)-\left(L_{11}\right)_{-}(s)=\eta_{L}(-s) L_{12}(s), s \in(-\infty, 0), \\
& L_{11}(s)=1+o(1), s \rightarrow \infty, \\
& L_{11}(s)=O\left(\epsilon_{\beta}(s)\right), s \rightarrow 0 .
\end{aligned}
$$

Hence, in view of the behavior of $L_{12}$; cf. (44)-(5), we have

$$
L_{11}(s)=1+\frac{1}{2 \pi i} \int_{-\infty}^{0} \frac{\eta_{L}(-\tau) L_{12}(\tau) d \tau}{\tau-s}=1+\frac{1}{2 \pi i} \int_{0}^{\infty} \frac{\eta_{L}(\tau) L_{12}(-\tau) d \tau}{-\tau-s}
$$

for $s \in \mathbb{C} \backslash(-\infty, 0]$, especially for $s \in(0, \infty)$. Similarly, it is also seen from (2) that $L_{12}(s)$ is analytic in $\mathbb{C} \backslash[0, \infty)$, and solves the scalar $\mathrm{RH}$ problem

$$
\begin{aligned}
& \left(L_{12}\right)_{+}(s)-\left(L_{12}\right)_{-}(s)=-\eta_{L}(s) L_{11}(s), s \in(0, \infty), \\
& L_{12}(s)=o(1), s \rightarrow \infty \\
& L_{12}(s)=O\left(\epsilon_{\beta}(s)\right), s \rightarrow 0 .
\end{aligned}
$$

Hence, we have

$$
L_{12}(s)=\frac{1}{2 \pi i} \int_{0}^{\infty} \frac{-\eta_{L}(\tau) L_{11}(\tau) d \tau}{\tau-s} \text { for } s \in \mathbb{C} \backslash[0, \infty),
$$

especially for $s \in(-\infty, 0)$. For $x \in(0,+\infty)$, now we define

$$
u_{\beta}(x)=L_{11}(x)+(-1)^{n} L_{12}(-x), \quad v_{\beta}(x)=L_{11}(x)-(-1)^{n} L_{12}(-x) .
$$

From (6)-(7) and (8), it is readily verified that $u_{\beta}$ and $v_{\beta}$ solve, respectively, the following integral equations

$$
\begin{array}{ll}
u(x)=1+\int_{0}^{\infty} \frac{K(t) u(t) d t}{t+x}, & x \in(0, \infty), \\
v(x)=1-\int_{0}^{\infty} \frac{K(t) v(t) d t}{t+x}, & x \in(0, \infty),
\end{array}
$$

where

$$
\begin{aligned}
K(t) & =\frac{1}{2 \pi i}\left[\exp \left(e^{\left(\frac{\pi}{2}+\frac{\beta \pi}{2}\right) i} t^{\beta}\right)-\exp \left(e^{-\left(\frac{\pi}{2}+\frac{\beta \pi}{2}\right) i} t^{\beta}\right)\right] \\
& =\frac{1}{\pi} \exp \left(-t^{\beta} \sin \frac{\pi \beta}{2}\right) \sin \left(t^{\beta} \cos \frac{\pi \beta}{2}\right)
\end{aligned}
$$


and $\eta_{L}(t)=2 \pi i(-1)^{n+1} K(t)$ for $t \in[0,+\infty)$.

It is worth noting that similar coupled scalar integral equations have been derived in [4], though the equations in that paper were used only to construct a contraction mapping. Also, it is mentioned in Fokas et al. [6, p.161] that a function $u(x)$ can be parametrized via the solution of a linear integral equation. This is exactly what we are doing: We are defining a pair of new special functions, using the above integral equations, to construct a parametrix.

Conversely, if $u_{\beta}(x)$ and $v_{\beta}(x)$ do solve the integral equations, with $u_{\beta}-1, v_{\beta}-1 \in L_{2}[0, \infty)$, then we can deduce that $u_{\beta}(z), v_{\beta}(z)=1+O(1 / z)$ as $z \rightarrow \infty$ for $|\arg z|<3 \pi / 2$ and are of the order $O\left(\epsilon_{\beta}(z)\right)$ as $z \rightarrow 0$ for $|\arg z| \leq \pi$; see the discussion in the next section, and especially Lemma 2. Thus, we have

Theorem 1. The piece-wise analytic function

$$
L(s)=\left\{\begin{array}{cc}
\left(\begin{array}{cc}
L_{\beta}(s) & (-1)^{n} U_{\beta}\left(s e^{-\pi i}\right) \\
(-1)^{n} U_{\beta}(s) & L_{\beta}\left(s e^{-\pi i}\right)
\end{array}\right), \quad 0<\arg s<\pi ; \\
\left(\begin{array}{cc}
L_{\beta}(s) & (-1)^{n} U_{\beta}\left(s e^{\pi i}\right) \\
(-1)^{n} U_{\beta}(s) & L_{\beta}\left(s e^{\pi i}\right)
\end{array}\right), \quad-\pi<\arg s<0
\end{array}\right.
$$

solves the RH problem (2), (4) and (5), where

$$
L_{\beta}(z)=\frac{1}{2}\left(u_{\beta}(z)+v_{\beta}(z)\right), \quad U_{\beta}(z)=\frac{1}{2}\left(u_{\beta}(z)-v_{\beta}(z)\right), \quad \arg z \in(-\infty, \infty), \quad z \neq 0 .
$$

\section{Unique solvability of the integral equations}

In [8], the unique solvability of the model problem for $L$ is justified in the Wiener class; i.e., the Fourier transform of each entry is in $L_{1}(\mathbb{R})$.

We note that in view of (14) the unique solvability of the integral equations (9)-(10) can accordingly be deduced from that of $L$.

Now we take an alternative approach, using the integral equations alone. We first show that both $u_{\beta}(x)-1$ and $v_{\beta}(x)-1$ are in $L_{2}[0,+\infty)$. We begin with the following lemma:

Lemma 1. The operator $T$ is a compact operator on $L_{2}[0,+\infty)$, where

$$
(T u)(x)=\int_{0}^{\infty} \frac{K(t) u(t) d t}{t+x} .
$$

Proof. The lemma is easily justified by noting that $\frac{K(t)}{t+x} \in L_{2}([0,+\infty) \times[0,+\infty))$. Thus, $T$ is a Hilbert-Schmidt operator, and hence is compact; cf. e.g. [16, p.277].

The next step is to show that the operator $I-T$ has trivial null space. To this aim, we need to know more about the analytic structure of the solutions to (9) and (10). Indeed, if $u(x)$ solves (9), then we see from equation (9) that $u$ can be extended analytically to $\mathbb{C} \backslash(-\infty, 0]$, and that $u(z)=1+O(1 / z)$ in subsectors. From (11), we see that the kernel $K(t)$ can be extended analytically to complex $t$ with $\arg t \in(-\infty, \infty)$, and is exponentially small for $|\arg t|<\pi / 2$. Using Cauchy's theorem, it can be shown that

$$
u(z)=1+\int_{0}^{\infty} \frac{K\left(t e^{i \theta}\right) u\left(t e^{i \theta}\right) d t}{t+e^{-i \theta} z}, \quad|\theta|<\frac{\pi}{2},
$$


which enables us to extend $u(z)$ to the larger sector $|\arg z|<3 \pi / 2$, and

$$
u(z)=1+O(1 / z), \quad \text { as } z \rightarrow \infty, \quad|\arg z|<3 \pi / 2 .
$$

Similar results can be obtained for $v(z)$. This procedure can be continued, since by using the Plemelj formula we have from (9)-(10)

$$
u\left(z e^{-\pi i}\right)-u\left(z e^{\pi i}\right)=2 \pi i K(z) u(z), \quad v\left(z e^{-\pi i}\right)-v\left(z e^{\pi i}\right)=-2 \pi i K(z) v(z)
$$

initially for real and positive $z$, and then for complex $z$. Appealing to the formulas in (18), we see that the above process of analytic continuation can keep on going until we have the results valid for $\arg z \in \mathbb{R}$. For later use, we need the following behavior of $(T u)(z)$ at the origin:

Lemma 2. For $u \in L_{2}[0,+\infty)$, we have $(T u)(z)=O\left(\epsilon_{\beta}(z)\right)$ for $|\arg z| \leq \pi$ as $z \rightarrow 0$; cf. (5), where $\epsilon_{\beta}(z)= \begin{cases}1, & 1 / 2<\beta<1, \\ \ln |z|, & \beta=1 / 2, \\ |z|^{\beta-1 / 2}, & 0<\beta<1 / 2 .\end{cases}$

Proof. Recall the function $K(t)$ in (12). For real and positive $z$, we have by using Hölder's inequality and splitting the interval of integration

$$
|(T u)(z)|^{2} \leq\|u\|_{2}^{2} \int_{0}^{\infty} \frac{K(t)^{2} d t}{(t+z)^{2}}=O\left(\int_{0}^{1} \frac{t^{2 \beta} d t}{(t+z)^{2}}\right)+O(1)=O\left(\epsilon_{\beta}(z)\right)
$$

as $z \rightarrow 0$. The last equality can be obtained by calculating a residue. With slight modifications, and taking into account the rotation formula (16), we can extend the above inequality to $|\arg z| \leq \pi$.

We show that the operator $I-T$ has trivial null space, by using a vanishing lemma technique. Similar argument can be found in, e.g., [8].

Lemma 3. Assume that $u \in L_{2}[0,+\infty)$ and $u-T u=0$ in $L_{2}[0,+\infty)$. Then it holds $u \equiv 0$ for $x \in[0, \infty)$ in $L_{2}[0,+\infty)$.

Proof. Indeed, from $u=T u$ we see that the above analytic continuation procedure works also for the present $u(z)$, the connection formula (18) still holds, and instead of (17) we have $u(z)=O(1 / z)$ as $z \rightarrow \infty$ for $|\arg z| \leq \pi$. We introduce an auxiliary vector function

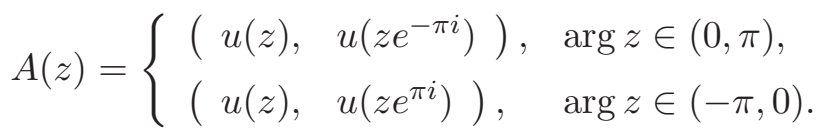

It is readily seen that $A(z)$ is analytic in both the upper and lower half plane, behaves uniformly as $O(1 / z)$ at infinity, and has jumps along the real axis as

$$
A_{+}(x)=A_{-}(x) J_{A}(x), \quad J_{A}(x)=\left\{\begin{array}{cc}
\left(\begin{array}{cc}
1 & 2 \pi i K(x) \\
0 & 1
\end{array}\right) & \text { for } x \in(0,+\infty), \\
\left(\begin{array}{cc}
1 & 0 \\
-2 \pi i K(|x|) & 1
\end{array}\right) & \text { for } x \in(-\infty, 0)
\end{array}\right.
$$

cf. (18). 
Denote by $A^{*}$ the conjugate transpose of $A$. Since $A_{+} A_{-}^{*}$ has an analytic continuation to the upper half plane, and $A_{-} A_{+}^{*}$ to the lower half, in view of the behavior $A(z)=O(1 / z)$ at infinity, by the Cauchy integral theorem we have

$$
\begin{aligned}
\int_{\mathbb{R}} A_{-}(x) J_{A}(x) A_{-}^{*}(x) d x & =\int_{\mathbb{R}} A_{+}(x) A_{-}^{*}(x) d x=0, \\
\int_{\mathbb{R}} A_{-}(x) J_{A}^{*}(x) A_{-}^{*}(x) d x & =\int_{\mathbb{R}} A_{-}(x) A_{+}^{*}(x) d x=0 .
\end{aligned}
$$

Here, use has also been made of the fact that $u(z)=O\left(\epsilon_{\beta}(z)\right)$ for small $z$ with $|\arg z| \leq \pi$; see Lemma 2. Summing up the integrals gives

$$
\int_{\mathbb{R}} A_{-}(x)\left(J_{A}(x)+J_{A}^{*}(x)\right) A_{-}^{*}(x) d x=0 .
$$

It can be seen from (12) that $|\pi i K(|x|)|<1$ for $x \in \mathbb{R}$. Hence,

$$
J_{A}(x)+J_{A}^{*}(x)=\left(\begin{array}{cc}
2 & 2 \pi i K(|x|) \\
-2 \pi i K(|x|) & 2
\end{array}\right)
$$

is positive definite for $x \in \mathbb{R}$. From (19) one deduces that $A_{-}(x) \equiv 0$, and thus $u(x) \equiv 0$ for $x \in(0,+\infty)$.

We note that $\int_{0}^{\infty} \frac{K(t) d t}{t+x} \in L_{2}[0,+\infty)$ since it is smooth on $(0, \infty)$, bounded at $0^{+}$, and of order $O(1 / x)$ at $+\infty$. Hence, a combination of Lemmas 1 and 3 gives the unique solvability of the following integral equation

$$
u_{1}(x)-T u_{1}(x)=\int_{0}^{\infty} \frac{K(t) d t}{t+x}
$$

in the space $L_{2}[0,+\infty)$. Similar analysis can be carried out for the other equation (10). To conclude, we have the following:

Theorem 2. There exist unique solutions $u(x)$ and $v(x)$ to the integral equations (9) and (10), respectively, such that $u(x)-1 \in L_{2}[0,+\infty)$ and $v(x)-1 \in L_{2}[0,+\infty)$.

\section{Boundedness of $u_{\beta}(x)$ and $v_{\beta}(x)$ on $[0, \infty)$}

The main result in the last section is that $u_{\beta}(x)-1 \in L_{2}[0,+\infty)$. By using successive approximation, one can actually show that $u_{\beta}(x)$ is continuous and bounded for $\beta>\beta_{c}:=0.4158853544$. Indeed, it suffices to show that the operator $T$ defined in (15) is a contraction on the space $L_{\infty}$ for those $\beta$. Straightforward estimation gives

$$
|(T u)(x)| \leq M_{\beta} \max _{[0,+\infty)}|u(t)| \text { for } x \in[0,+\infty), \text { with } M_{\beta}=\int_{0}^{\infty} \frac{|K(t)| d t}{t} \leq \frac{\cot \left(\frac{\pi \beta}{2}\right)}{\pi \beta} .
$$

The bound $\cot \left(\frac{\pi \beta}{2}\right) / \pi \beta$ is monotonically decreasing for $\beta \in(0,1)$, and is less than 1 for $\beta>\beta_{c}$.

In this section, we shall show that $u_{\beta}(x)$ and $v_{\beta}(x)$ are in fact bounded for all $\beta \in(0,1)$.

Theorem 3. Let $u_{\beta}(x)$ and $v_{\beta}(x)$ be solutions of the integral equations in (9) and (10), respectively. Then, $u_{\beta}(x), v_{\beta}(x) \in L_{\infty}[0, \infty)$ and $u_{\beta}(x), v_{\beta}(x) \in C[0, \infty)$. 
Proof. Let $\tilde{u}(x)=u_{\beta}(x)-1$. By Theorem 2, $\tilde{u}(x) \in L_{2}[0, \infty)$. Rewrite equation (9) as

$$
\tilde{u}(x)=\int_{0}^{\infty} \frac{K(t) \tilde{u}(t) d t}{t+x}+u_{0}(x), \quad x \in[0, \infty),
$$

where

$$
u_{0}(x)=\int_{0}^{\infty} \frac{K(t)}{t+x} d t
$$

Clearly, $u_{0}(x) \in L_{2}[0, \infty) \cap L_{\infty}[0, \infty)$. The function $\tilde{u}(x)$ is smooth in $(0, \infty)$, since it is an analytic function in the cut plane $\mathbb{C} \backslash(-\infty, 0]$. To prove that $\tilde{u}(x)$ is also bounded there, we divide our discussion into several cases. In general, for all $\beta \in(0,1)$, we have

$$
|\tilde{u}(x)| \leq\left|u_{0}(x)\right|+\int_{0}^{\infty} \frac{|K(t)||\tilde{u}(t)| d t}{t+x} \leq \frac{1}{x} \int_{0}^{\infty}|K(t)|(1+|\tilde{u}(t)|) d t \leq \frac{1}{x}\left(\|K\|_{1}+\|K\|_{2}\|\tilde{u}\|_{2}\right)
$$

for all positive $x$, and especially for $x \geq 1$. We may focus on $0<x \leq 1$.

Case (i) $\frac{1}{2}<\beta<1$. For $0<x \leq 1$, we have

$$
|\tilde{u}(x)| \leq a+\int_{0}^{\infty} \frac{|K(t)||\tilde{u}(t)|}{t+x} d t \leq a+\int_{0}^{\infty} \frac{|K(t)|}{t}|\tilde{u}(t)| d t \leq a+\left\|\frac{K(t)}{t}\right\|_{2}\|\tilde{u}\|_{2},
$$

where $a=\left\|u_{0}\right\|_{\infty}$. Thus, $\tilde{u}(x)$ is bounded. Here, we have used the fact that $\frac{K(t)}{t} \in L_{2}[0, \infty)$.

Case (ii) $\frac{1}{4}<\beta \leq \frac{1}{2}$. In this case, we write $\beta=\frac{1}{4}+\varepsilon$ with $\varepsilon \in\left(0, \frac{1}{4}\right]$. We first recall the inequality [10, p.30]

$$
x^{\frac{1}{p}} y^{\frac{1}{q}} \leq \frac{x}{p}+\frac{y}{q} \leq x+y,
$$

where $x \geq 0, y \geq 0$ and $1 / p+1 / q=1$ with $p>1$. From this inequality, we have

$$
t+x \geq t^{\beta+\frac{1}{2}-\frac{\varepsilon}{2}} x^{\frac{1}{2}-\beta+\frac{\varepsilon}{2}} .
$$

Note that $\beta+\frac{1}{2}-\frac{\varepsilon}{2}=\frac{3}{4}+\frac{\varepsilon}{2}>0$ and $\frac{1}{2}-\beta+\frac{\varepsilon}{2} \geq \frac{\varepsilon}{2}>0$. Consequently, we have

$$
|\tilde{u}(x)| \leq a+\int_{0}^{\infty} \frac{|K(t)||\tilde{u}(t)|}{t+x} d t \leq a+\left\|\frac{K(t)}{t^{\beta+\frac{1}{2}-\frac{\varepsilon}{2}}}\right\|_{2}\|\tilde{u}\|_{2} \frac{1}{x^{\frac{1}{2}-\beta+\frac{\varepsilon}{2}}} \leq \frac{c}{x^{\frac{1}{2}-\beta+\frac{\varepsilon}{2}}}, \quad x \in(0,1],
$$

where $c$ is a generic positive constant. The fact that $K(t) / t^{\beta+\frac{1}{2}-\frac{\varepsilon}{2}} \in L_{2}[0, \infty)$ is readily justified by its behavior $O\left(t^{\frac{\varepsilon}{2}-\frac{1}{2}}\right)$ at $t=0^{+}$, and its exponential decay at $t=+\infty$. This estimate can be used to self-improve and achieve

$$
|\tilde{u}(x)| \leq a+\int_{0}^{\infty} \frac{|K(t)||\tilde{u}(t)|}{t} d t \leq a+\left\|\frac{K(t)}{t^{1-\beta+\varepsilon}}\right\|_{2}\left\|t^{-\beta+\varepsilon} \tilde{u}(t)\right\|_{2}
$$

for $x \in(0, \infty)$. To see that the two quantities in the last term of the above inequality are finite, we just check their behavior as $t \rightarrow 0^{+}$and as $t \rightarrow+\infty$. For instance, as $t \rightarrow 0^{+}$, we have $K(t) / t^{1-\beta+\varepsilon}=O\left(t^{2 \beta-1-\varepsilon}\right)=O\left(t^{\varepsilon-\frac{1}{2}}\right)$ since $\beta=\frac{1}{4}+\varepsilon$ and $t^{-\beta+\varepsilon} \tilde{u}(t)=O\left(t^{\frac{\varepsilon}{2}-\frac{1}{2}}\right)$ by the estimate in $(22)$. 
Case (iii) $\frac{1}{2(k+1)}<\beta \leq \frac{1}{2 k}, k=2,3, \cdots$. The analysis is very much the same as that given for the previous case, except that we need to repeat the self-improvement argument several times. First, let us write $\beta=\frac{1}{2(k+1)}+\varepsilon$ with $\varepsilon \in\left(0, \frac{1}{2 k(k+1)}\right]$. Straightforward calculation shows

$$
k \beta+\frac{1}{2}-\frac{(2 k-1) \varepsilon}{2}>\cdots>2 \beta+\frac{1}{2}-\frac{3 \varepsilon}{2}>\beta+\frac{1}{2}-\frac{\varepsilon}{2}=\frac{1}{2(k+1)}+\frac{1}{2}+\frac{\varepsilon}{2}>0,
$$

and

$$
\frac{1}{2}-\beta+\frac{\varepsilon}{2}>\frac{1}{2}-2 \beta+\frac{3 \varepsilon}{2}>\cdots>\frac{1}{2}-k \beta+\frac{(2 k-1) \varepsilon}{2}=\frac{1}{2(k+1)}-\frac{\varepsilon}{2} \geq \frac{2 k-1}{4 k(k+1)}>0 .
$$

Hence, for $x \in(0,1]$, repeated application of the inequality in (20) and the self-improvement steps in (21) and (22) lead to

$$
\begin{aligned}
& \left\{\begin{array}{l}
t+x \geq t^{\beta+\frac{1}{2}-\frac{\varepsilon}{2}} x^{\frac{1}{2}-\beta+\frac{\varepsilon}{2}} \text { and } \\
|\tilde{u}(x)| \leq a+\left\|\frac{K(t)}{t^{\beta+\frac{1}{2}-\frac{\varepsilon}{2}}}\right\|_{2}\|\tilde{u}(t)\|_{2} \frac{1}{x^{\frac{1}{2}-\beta+\frac{\varepsilon}{2}}} \leq \frac{c}{x^{\frac{1}{2}-\beta+\frac{\varepsilon}{2}}},
\end{array}\right. \\
& \left\{\begin{array}{l}
t+x \geq t^{2 \beta+\frac{1}{2}-\frac{3 \varepsilon}{2}} x^{\frac{1}{2}-2 \beta+\frac{3 \varepsilon}{2}} \quad \text { and } \\
|\tilde{u}(x)| \leq a+\left\|\frac{K(t)}{t^{\beta+\frac{1}{2}-\frac{\varepsilon}{2}}}\right\|_{2}\left\|t^{-\beta+\varepsilon} \tilde{u}(t)\right\|_{2} \frac{1}{x^{\frac{1}{2}-2 \beta+\frac{3 \varepsilon}{2}}} \leq \frac{c}{x^{\frac{1}{2}-2 \beta+\frac{3 \varepsilon}{2}}}
\end{array}\right. \\
& \left\{\begin{array}{l}
t+x \geq t^{k \beta+\frac{1}{2}-\frac{(2 k-1) \varepsilon}{2}} x^{\frac{1}{2}-k \beta+\frac{(2 k-1) \varepsilon}{2}} \text { and } \\
|\tilde{u}(x)| \leq a+\left\|\frac{K(t)}{t^{\beta+\frac{1}{2}-\frac{\varepsilon}{2}}}\right\|_{2}\left\|t^{-(k-1)(\beta-\varepsilon)} \tilde{u}(t)\right\|_{2} \frac{1}{x^{\frac{1}{2}-k \beta+\frac{(2 k-1) \varepsilon}{2}}} \leq \frac{c}{x^{\frac{1}{2}-k \beta+\frac{(2 k-1) \varepsilon}{2}}} .
\end{array}\right.
\end{aligned}
$$

The last estimate ensures the finiteness of the two $L^{2}$-norms in the following inequalities

$$
|\tilde{u}(x)| \leq a+\int_{0}^{\infty} \frac{|K(t)||\tilde{u}(t)|}{t} d t \leq a+\left\|\frac{K(t)}{t^{1-k \beta+k \varepsilon}}\right\|_{2}\left\|t^{-k \beta+k \varepsilon} \tilde{u}(t)\right\|_{2} .
$$

This establishes the boundedness of $u_{\beta}(x)$ for $x \in(0,1]$, and hence also for $x \in(0, \infty)$. Since $u_{\beta}(x)$ is bounded on $(0, \infty)$, the continuity of $u_{\beta}(x)$ at $x=0$ from the right now follows from equation (9) and the Lebesgue dominated convergence theorem.

An analogous argument gives $v_{\beta}(x) \in L_{\infty}[0, \infty)$ and $v_{\beta}(x) \in C[0, \infty)$.

\section{$5 \quad$ Asymptotic behavior and Stokes phenomenon}

From the integral equations, and in view of (16), we readily obtain the asymptotic approximations

$$
u_{\beta}(z) \sim 1+\sum_{k=1}^{\infty} \frac{c_{k}}{z^{k}} \text { and } v_{\beta}(z) \sim 1+\sum_{k=1}^{\infty} \frac{d_{k}}{z^{k}} \text { as }|z| \rightarrow+\infty, \quad|\arg z|<\frac{3 \pi}{2},
$$

where

$$
c_{k}=(-1)^{k-1} \int_{0}^{\infty} K(t) t^{k-1} u_{\beta}(t) d t \text { and } d_{k}=(-1)^{k} \int_{0}^{\infty} K(t) t^{k-1} v_{\beta}(t) d t
$$

for $k=1,2, \cdots$; cf. Boyd [1] for asymptotic approximations of Stieltjes transforms. See also Wong [12], and Wong and Zhao [14]. 
To satisfy certain jump conditions and matching conditions, the Stokes phenomenon plays a crucial role in the construction of local parametrices for RHPs. Now we turn to a brief discussion of the Stokes phenomena of $u_{\beta}$ and $v_{\beta}$, based on the connection formulas in (18), and regarding equations (91)-(10) as exact resurgence relations. A similar analysis was carried out in Boyd [1] for the modified Bessel function; see also Xu and Zhao [15] for an attempt to link together the $\mathrm{RH}$ approach with resurgence properties.

Assume that $u(x)=u_{\beta}(x)$ solves the integral equation (9) with $u-1 \in L_{2}[0, \infty)$, and $u(z)$ denotes its analytic continuation. In general, we see that $u(z)=1+O(1 / z)$ for large $z$ as $|\arg z|<\frac{3 \pi}{2}$; cf. (17). Coupling (17) and (18), one can verify that

$$
u(z)=1+O\left(\frac{1}{z}\right)+\exp \left(e^{-i \pi(1+\beta) / 2}\left(z e^{-\pi i}\right)^{\beta}\right)
$$

for $\frac{\pi}{2}<\arg z \leq \frac{3 \pi}{2}$; see also (11). The term on the extreme right is exponentially large, when $\arg z$ goes beyond $\frac{3 \pi}{2}$. According to Berry's Stokes smoothing, the switch-on of the exponential occurs when $1+O(1 / z)$ is the most dominant as compared with the exponential term, namely, when $-\left[\frac{\pi}{2}+\left(\frac{3 \pi}{2}-\arg z\right) \beta\right]=-\pi$, i.e., $\arg z=\frac{3 \pi}{2}-\frac{\pi}{2 \beta}$. We give some details in what follows.

In view of (11), we may write

$$
u(z)=1+\frac{1}{2 \pi i} \int_{0}^{\infty} \frac{e^{i e^{\beta \pi i / 2} t^{\beta}} u(t) d t}{t+z}-\frac{1}{2 \pi i} \int_{0}^{\infty} \frac{e^{-i e^{-\beta \pi i / 2} t^{\beta}} u(t) d t}{t+z}:=1+I_{P}-I_{N},
$$

initially for real positive $z$, and then extended elsewhere. Now we assume that $\beta \in[1 / 3,1)$, and take $I_{N}(z)$ as an example. Rotating the integration path clockwise to $\arg t=\phi \in[-\pi, 0)$, and making a change of variables $t=e^{-\pi i} z \tau^{\alpha}$ with $\alpha=1 / \beta$, we have

$$
I_{N}(z)=\frac{\alpha}{2 \pi i} \int_{0}^{\infty} \frac{e^{-\rho e^{i \theta} \tau} u\left(e^{-\pi i} z \tau^{\alpha}\right) \tau^{\alpha-1} d \tau}{\tau^{\alpha}-1}
$$

where $\theta=\beta\left(\arg z-\frac{\pi}{2}(3-\alpha)\right)$, the large parameter is $\rho=|z|^{\beta}$, and the path of integration is indented to pass above the pole at $\tau=1$. Before reaching the equality in (25), we have already rotated the path $\arg \tau=\phi+\pi$ to the positive real line.

Substitute the identity $\frac{1}{\tau^{\alpha}-1}=-\sum_{k=1}^{N-1} \tau^{k \alpha}+\frac{\tau^{N \alpha}}{\tau^{\alpha}-1}$ into the integral in (25). The remainder in the resulting expansion is an integral with a saddle point as well as a pole. Optimal truncation of the expansion occurs when the saddle point coalesces with the pole (see [1, 13]); that is, when $\rho \approx N \alpha$ or, more precisely, $\alpha N=\rho+r$, with $r$ being bounded. The result we have now is an exponentially improved asymptotic expansion, whose error term is given by

$$
\frac{1}{2 \pi i} \int_{0}^{\infty}\left\{\frac{\tau-1}{\tau^{\alpha}-1} u\left(e^{-\pi i} z \tau^{\alpha}\right) \alpha \tau^{\alpha-1+r}\right\} \frac{e^{-\rho\left(e^{i \theta} \tau-\ln \tau\right)} d \tau}{\tau-1},
$$

which exhibits a Stokes smoothing of error-function type as $\theta \rightarrow 0^{-}$when the saddle point $\tau=e^{-i \theta}$ coalesces with the simple pole $\tau=1$. This indicates that in our case a Stokes line occurs when we have $\theta=0$, i.e., $\arg z=\frac{\pi}{2}(3-\alpha)$. The analysis for the other integral $I_{P}(z)$ gives another Stokes line $\arg z=-\frac{\pi}{2}(3-\alpha)$.

A slight modification is needed for the case $\beta \in[1 / 5,1 / 3)$, since $\phi_{0}=-\frac{\pi}{2}(\alpha-1) \in[-2 \pi,-\pi)$ in this case. Rotating the integration path clockwise and picking up the residue $r_{N}(z)=$ $-\exp \left(e^{-\left(\frac{\pi}{2}+\frac{\beta \pi}{2}\right) i}\left(z e^{-\pi i}\right)^{\beta}\right) u\left(z e^{-\pi i}\right)$ at $t=z e^{-\pi i}$, one has

$$
I_{N}(z)=r_{N}+\frac{1}{2 \pi i} \int_{0}^{\infty e^{i \phi}} \frac{e^{-i e^{-\frac{\beta \pi i}{2}} t^{\beta}} u(t) d t}{t+z}=J_{N}(z)+\frac{1}{2 \pi i} \int_{0}^{\infty e^{i \phi}} \frac{e^{-i e^{-\frac{\beta \pi i}{2}} t^{\beta}} u\left(t e^{2 \pi i}\right) d t}{t+z}
$$


where $\phi<-\pi$, and $J_{N}(z)$ is a sum of exponentials and integrals. The second equality is obtained by substituting in $u(t)=u\left(t e^{2 \pi i}\right)+K\left(t e^{\pi i}\right) u\left(t e^{\pi i}\right)$; cf. (18). Now we focus on the last integral. Making a change of variables $t=e^{-\pi i} z \tau^{\alpha}$, and using the same optimal truncation $\alpha N=\rho+r$ with $\rho=|z|^{\beta}$, we obtain a slightly different error term

$$
\frac{1}{2 \pi i} \int_{0}^{\infty}\left\{\frac{\tau-1}{\tau^{\alpha}-1} u\left(e^{\pi i} z \tau^{\alpha}\right) \alpha \tau^{\alpha-1+r}\right\} \frac{e^{-\rho\left(e^{i \theta} \tau-\ln \tau\right)} d \tau}{\tau-1},
$$

where again $\theta=\beta\left(\arg z-\frac{\pi}{2}(3-\alpha)\right)$, the large parameter $\rho=|z|^{\beta}$, but the path of integration is indented to pass below the pole at $\tau=1$. As before, a Stokes line in this case is identified as $\arg z=-\frac{\pi}{2}(\alpha-3) \in(-\pi, 0)$. Another symmetric Stokes line $\arg z=\frac{\pi}{2}(\alpha-3)$ can be found by analyzing $I_{P}(z)$; see (24).

In general, for $\beta \in\left[\frac{1}{4 l+3}, \frac{1}{4 l-1}\right), l=1,2, \cdots$, repeated use of the connection formula (18) gives

$$
I_{N}(z)=J_{N}(z)+\frac{1}{2 \pi i} \int_{0}^{\infty e^{i \phi}} \frac{e^{-i e^{-\frac{\beta \pi i}{2}} t^{\beta}} u\left(t e^{2 l \pi i}\right) d t}{t+z}
$$

where $\phi<-(2 l-1) \pi$. Here again $J_{N}(z)$ is a combination of exponentials and integrals. Similar analysis can be carried out for the last integral to locate the Stokes line. The same treatment also works for $v_{\beta}$. The reader is referred to [13, p.360] for full details.

To summarize, we have

Theorem 4. Assume that the functions $u_{\beta}(z)$ and $v_{\beta}(z)$, solving respectively (9) and (10), such that $u_{\beta}-1, v_{\beta}-1 \in L_{2}[0, \infty)$. Then the Stokes lines for both functions are

$$
\arg z= \pm\left\{\frac{\pi}{2}(3-\alpha)+2(l-1) \pi\right\}
$$

where $\alpha=\frac{1}{\beta} \in(4 l-3,4 l+1], l=1,2, \cdots$.

\section{Discussion}

In the previous sections, we have determined a pair of new special functions $u_{\beta}$ and $v_{\beta}$ via integral equations. Preliminary analysis reveals some of the analytic structures of the functions, such as their analytic continuations, connection formulas, and asymptotic results involving Stokes phenomenon. Still we have the following open problems.

Problem 1. Here we propose a thorough investigation of the analytic and asymptotic properties of the functions $u_{\beta}$ and $v_{\beta}$, such as their zeros, modulus function [11, §10.18], kernel [9], and differential or difference equations, etc. For example, one may begin with

(i) Determine the coefficients $c_{k}$ and $d_{k}$ of the expansion at infinity given in (23), which are now expressed in terms of $u_{\beta}$ and $v_{\beta}$. It would be interesting and challenging to decode the coefficients from (9) and (10) in an explicit manner;

(ii) On account of (29), one may reasonably expect that the behaviors of $u_{\beta}$ and $v_{\beta}$ at the origin are of the form $\sum_{k=0} \tilde{c}_{k} z^{k}+\sum_{k=1} \tilde{d}_{k} z^{\beta k}$; see also Theorem 3 and Remark 1 for the boundedness of these functions. A natural problem is to determine explicitly the coefficients $\tilde{c}_{k}$ and $\tilde{d}_{k}$, at least for $k=0$ and 1 . 
The Stieltjes transform of the function $K(t)$ given in (11) suggests that it would also be of interest to study the related integral

$$
G_{\beta}(z):=\int_{0}^{\infty} \frac{e^{-t^{\beta}}}{t+z} d t
$$

for $\beta>0$, defined initially for $\arg z \in(-\pi, \pi)$ and then analytically continued to $\arg z \in \mathbb{R}$. The special case when $\beta=2$ is termed the Goodwin-Staton integral [11, (7.2.12)]; see also Jones [7], where, by making use of the incomplete gamma function, asymptotic formulas are obtained for a slightly more general integral.

The Mittag-Leffler function can be represented in terms of this function. For $\alpha=1 / \beta>0$, the Mittag-Leffler function is given by

$$
E_{\alpha}(z):=\sum_{n=0}^{\infty} \frac{z^{n}}{\Gamma(\alpha n+1)}=\beta \sum e^{Z_{k}}+\frac{1}{2 \pi i} \int_{C} \frac{t^{\alpha-1} e^{t} d t}{t^{\alpha}-z}:=\Sigma(z)+I(z),
$$

where $Z_{k}=z^{\beta} e^{2 \pi i k \beta}$ for integer $k$ and the summation is taken over all $k$ satisfying $\left|\arg Z_{k}\right|<\pi$. The path $C$ is a Hankel contour, and dented properly if $\left|\arg Z_{k}\right|=\pi$ for a certain integer $k$; cf. [13.

As in Wong and Zhao [13], it is appropriate to divide our discussion into several cases: (i) $\alpha$ is a positive integer, (ii) $\alpha \in(2 l-1,2 l), l=1,2,3, \cdots$, and (iii) $\alpha \in(2 l, 2 l+1), l=0,1,2, \cdots$.

In case (i), i.e., when $\alpha$ is a positive integer, the contour integral in (27) vanishes; thus the function $E_{\alpha}(z)$ is an elementary function. In case (ii), we have

$$
I(z)=\frac{\beta}{2 \pi i} G_{\beta}\left(e^{(2 l-1-\alpha) \pi i} z\right)-\frac{\beta}{2 \pi i} G_{\beta}\left(e^{(\alpha-2 l+1) \pi i} z\right)
$$

initially for $\arg z=0$, and then extend the region of validity by analytic continuation. Similarly, in case (iii),

$$
I(z)=\frac{\beta}{2 \pi i} G_{\beta}\left(e^{(2 l+1-\alpha) \pi i} z\right)-\frac{\beta}{2 \pi i} G_{\beta}\left(e^{(\alpha-2 l-1) \pi i} z\right) .
$$

Asymptotic expansions of $G_{\beta}$ can be derived as follows. For example, at infinity, we have

$$
G_{\beta}(z) \sim \sum_{n=1}^{\infty} \frac{(-1)^{n-1} \Gamma(n / \beta)}{\beta z^{n}}
$$

initially for $|\arg z|<\pi$. The region of validity can be extended to $|\arg z|<\pi+\frac{\pi}{2 \beta}$. Also, at the origin, we write $G_{\beta}(z)=\left\{\int_{0}^{1}+\int_{1}^{\infty}\right\} \frac{e^{-t^{\beta}} d t}{t+z}$. Expanding the exponential in the first integral in convergent series, and using Cauchy residue theorem, we have

$$
G_{\beta}(z) \sim-\ln z+\sum_{n=1}^{\infty} \frac{(-1)^{n+1} \pi}{n ! \sin (\beta n \pi)} z^{\beta n}+\sum_{k=0}^{\infty} c_{\beta, k}(-1)^{k} z^{k}, \quad z \rightarrow 0
$$

so long as $\beta$ is not a rational number, where

$$
c_{\beta, 0}=\sum_{n=1}^{\infty} \frac{(-1)^{n}}{n ! n \beta}+\frac{\Gamma(0,1)}{\beta}=\frac{1}{\beta}\left[\int_{0}^{1} \frac{e^{-t}-1}{t} d t+\int_{1}^{\infty} \frac{e^{-t}}{t} d t\right]=-\frac{\gamma}{\beta}
$$


and

$$
c_{\beta, k}=\sum_{n=0}^{\infty} \frac{(-1)^{n}}{n !(n \beta-k)}+\frac{\Gamma(-k / \beta, 1)}{\beta}, \quad k=1,2, \cdots,
$$

where $\Gamma(a, z)=\int_{z}^{\infty} t^{a-1} e^{-t} d t$ is the incomplete Gamma function. For the last equality in (30), see [11, (6.2.1), (6.2.3)-(6.2.4)], $\gamma$ being Euler's constant. It can be shown that the expansion in (29) is valid in the sector $|\arg z|<\pi+\frac{\pi}{2 \beta}$, as long as $\beta$ is a positive irrational number.

If $\beta>0$ is a rational number, then we write $\beta=p / q$ with $p$ and $q$ being relatively prime. The change of variable $t^{1 / q}=s$ gives

$$
G_{\beta}(z)=\int_{0}^{\infty} \frac{q s^{q-1} e^{-s^{p}}}{s^{q}+\zeta^{q}} d \zeta, \quad \zeta^{q}=z .
$$

Let $\omega_{l}=e^{(2 l+1) \pi i / q}, l=0,1, \cdots, q-1$. Since

$$
s^{q}+\zeta^{q}=\left(s-\omega_{0} \zeta\right)\left(s-\omega_{1} \zeta\right) \cdots\left(s-\omega_{q-1} \zeta\right),
$$

taking logarithmic derivative yields

$$
\frac{q s^{q-1}}{s^{q}+\zeta^{q}}=\sum_{l=0}^{q-1} \frac{1}{s-\omega_{l} \zeta} .
$$

Thus, $G_{\beta}(z)$ in (31) can be written as

$$
G_{\beta}(z)=\sum_{l=0}^{q-1} G_{p}\left(e^{(2 l+1-q) \pi i / q} \zeta\right) .
$$

The problem is now reduced to consider the function $G_{p}(z)$, where $p$ is a positive integer. In this case, the integral for $G_{p}(z)$ can again be broken into two parts, one over the interval $(0,1)$ and the other on the infinite interval $(1, \infty)$. The integral on $(1, \infty)$ can be asymptotically evaluated as before. For the integral on $(0,1)$, we expand the exponential function $e^{-t^{p}}$ into a power series. This leads to infinitely many integrals of the form

$$
I_{n}(z)=\int_{0}^{1} \frac{t^{n p}}{t+z} d t, \quad n=0,1,2, \cdots .
$$

For each $n \geq 1$, we have

$$
\frac{1}{t+z}=\frac{1}{t}-\frac{z}{t^{2}}+\cdots+(-1)^{n p-1} \frac{z^{n p-1}}{t^{n p}}+(-1)^{n p} \frac{z^{n p}}{t^{n p}(t+z)},
$$

and hence

$$
I_{n}(z)=\sum_{k=0}^{n p-1} \frac{(-1)^{k} z^{k}}{n p-k}+(-1)^{n p} z^{n p}[\ln (1+z)-\ln z] .
$$

Asymptotic expansion of $G_{p}(z)$ can thus be obtained for small values of $z$.

Problem 2. We have used numerical simulation to draw the graphs of $u_{\beta}(x)$ for various values $\beta \in(0,1)$; see Figure 1. From the graphs, it is reasonable to conjecture that the function $u_{\beta}(x)$ is monotonically decreasing in $x \in[0, \infty)$, and also that the initial value $u_{\beta}(0)$ is decreasing in $\beta \in(0,1)$. What we need now are proofs of these statements. From Figure 1, corresponding conjectures can be made for the function $v_{\beta}(x)$. 

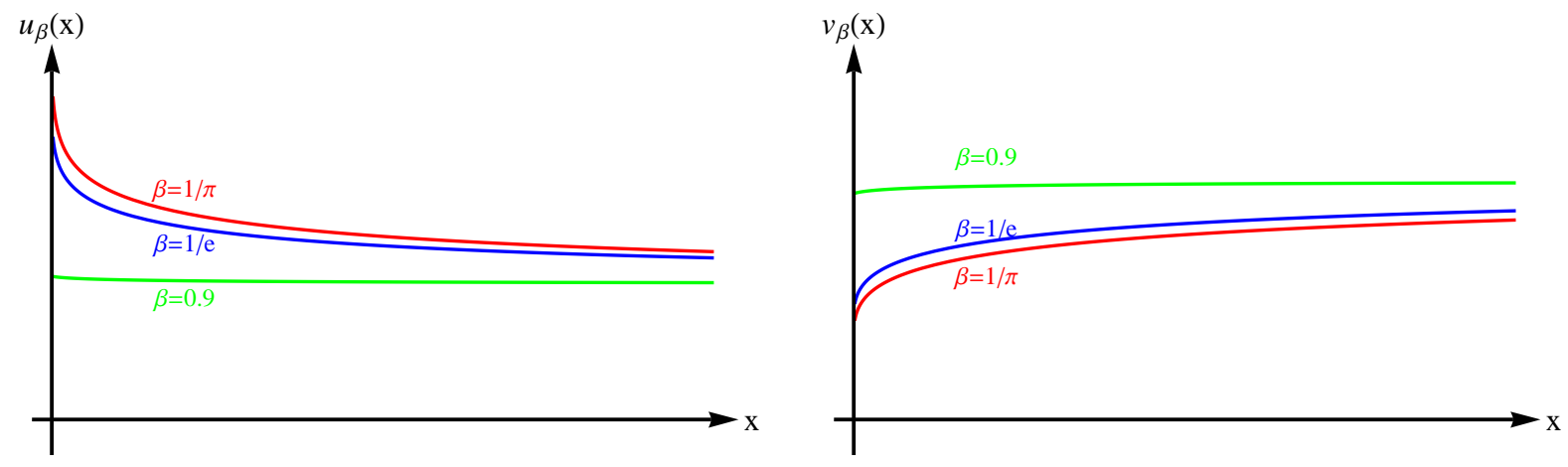

Figure 1: The solution profiles of $u_{\beta}(x)$ (left) and $v_{\beta}(x)$ (right).

\section{Acknowledgements}

We would like to thank Dr. Xiang-Sheng Wang for drawing the graphs and helping us with the numerical simulation. The research of Yu-Qiu Zhao was supported in part by the National Natural Science Foundation of China under grant numbers 10871212 and 11571375.

\section{References}

[1] W.G.C. Boyd, Stieltjes transforms and the Stokes phenomenon. Proc. Roy. Soc. London A, 429(1990), 227-246.

[2] Y. Chen and M. Ismail, Some indeterminate moment problems and Freud-like weights, Constr. Approx., 14(1998), 439-458.

[3] D. Dai, M. Ismail and X.-S. Wang, Plancherel-Rotach asymptotic expansion for some polynomials from indeterminate moment problems, Constr. Approx. 40(2014), 61-104.

[4] A. Deaño, A. Kuijlaars and P. Román, Asymptotic behavior and zero distribution of polynomials orthogonal with respect to Bessel functions, arXiv:1406.0969.

[5] P. Deift, T. Kriecherbauer, K.T.-R. McLaughlin, S. Venakides and X. Zhou, A RiemannHilbert approach to asymptotic questions for orthogonal polynomials, J. Comput. Appl. Math., 133(2001), 47-63.

[6] A.S. Fokas, A.R. Its, A.A. Kapaev and V.Y. Novokshenov, Painlevé transcendents. The Riemann-Hilbert approach, Mathematical Surveys and Monographs, 128, American Mathematical Society, Providence, RI, 2006.

[7] D.S. Jones, The generalized Goodwin-Staton integral, Proc. Edinb. Math. Soc., 48(2005), 635-650.

[8] T. Kriecherbauer and K.T.-R. McLaughlin, Strong asymptotics of polynomials orthogonal with respect to Freud weights, Int. Math. Res. Not., 1999(1999), 299-333.

[9] A.B.J. Kuijlaars and M. Vanlessen, Universality for eigenvalue correlations from the modified Jacobi unitary ensemble, Int. Math. Res. Not., 2002(2002), 1575-1600. 
[10] D.S. Mitrinović, Analytic inequalities, Springer-Verlag, Berlin, 1970.

[11] F. Olver, D. Lozier, R. Boisvert and C. Clark, NIST handbook of mathematical functions, Cambridge University Press, Cambridge, 2010.

[12] R. Wong, Asymptotic approximations of integrals, Academic Press, Boston, MA, 1989, reprinted (SIAM), Philadelphia, PA, 2001.

[13] R. Wong and Y.-Q. Zhao, Exponential asymptotics of the Mittag-Leffler function, Constr. Approx. 18(2002), 355-385.

[14] R. Wong and Y.-Q. Zhao, Gevrey asymptotics and Stieltjes transforms of algebraically decaying functions, Proc. Roy. Soc. London A 458(2002), 625-644.

[15] S.-X. Xu and Y.-Q. Zhao, Resurgence relation and global asymptotic analysis of orthogonal polynomials via the Riemann-Hilbert approach, Sci. China Math. 54(2011), 661-679.

[16] K. Yosida, Functional analysis, reprint of the sixth edition, Springer-Verlag, Berlin, 1995. 